\title{
Myomectomy of incision site uterine fibroid during caesarean section: a case report
}

\author{
Ashu Yadav, Naina Kumar \\ Correspondence: Dr. Naina Kumar, Associate Professor, Department of Obstetrics and \\ Gynaecology, All India Institute of Medical Sciences, Mangalagiri, Guntur, India; \\ Email - drashu102@gmail.com \\ Distributed under Attribution - NonCommercial - Share Alike 4.0 International (CC BY-NC-SA 4.0)
}

\begin{abstract}
Fibroids are common benign smooth muscle tumours occurring in $20-40 \%$ of women with an incidence of approximately $0.1 \%$ to $12.5 \%$ of all pregnancies. A 23 years old primigravida at 38 weeks 5 days gestational age referred from a private hospital with history of attempted emergency lower segment caesarean section in view of transverse lie under spinal anaesthesia and failed to deliver the baby and referred to our institute after closing the abdomen. On admission, ultrasound showed a large fibroid of $9 \times 8 \mathrm{~cm}^{2}$ on anterior wall of the lower segment of uterus. Abdomen was re-opened from previous incision site and a live baby delivered. Due to myoma the two ends of incised uterine wall could not be approximated, so myomectomy was planned. Histopathology confirmed leiomyoma.
\end{abstract}

Keywords: Lower segment caesarean section, myoma, ultrasonography.

Fibroids, also known as myomas are common benign smooth muscle tumours occurring in $20-40 \%$ of women, whereas the incidence rate in pregnancy varies between $0.1 \%$ to $12.5 \%{ }^{1}$. The incidence of fibroid increases with maternal age at pregnancy ${ }^{2}$. The exact etiology of uterine myomas is still not clear. During pregnancy the fibroids can be easily confused with other adnexal masses and its sonographic imaging is indispensable. Fibroid less than 5 $\mathrm{cm}$ in diameter tend to remain stable or decrease in size and larger fibroids $(>5 \mathrm{~cm})$ tend to grow during the pregnancy $^{2}$.

During pregnancy, uterine leiomyoma are usually asymptomatic but may sometimes leads to increased risk of adverse events like spontaneous abortion, preterm labor, premature rupture of membranes, antepartum hemorrhage, red degeneration, malpresentations, obstructed labour, caesarean section and postpartum hemorrhage ${ }^{3-5}$. Various studies revealed that $30 \%$ fibroids increase in volume, especially in first trimester and minimal during second and third trimester ${ }^{6}$.

Here we present a rare case report of term pregnancy with large fibroid uterus in which the patient was taken up for LSCS in view of transverse lie at some private hospital but was referred to our institute as the baby could not be extracted out.

\section{Case report}

A 23 years old primigravida at 38 week 5 days gestational age was referred from a private hospital to the

Received: $6^{\text {th }}$ March 2019. Accepted: $28^{\text {th }}$ May 2019.

Yadav A, Kumar N. Myomectomy of incision site uterine fibroid during caesarean section: a case report. The New Indian Journal of OBGYN. 2019; 6(1): 57-9. 
emergency department of a rural tertiary care centre of northern India with history of attempted emergency lower segment caesarean section under spinal anaesthesia in view of transverse lie, but as per the records, the surgeons failed to deliver the baby and referred the patient to our Institute after closing the abdomen. On examination the patient was well oriented to time, place and person, afebrile with pulse rate of 90 beats per minute and blood pressure 130/90mmHg, pallor and pedal oedema were present, respiratory and cardiovascular system examination revealed no abnormality. On per abdominal examination, uterine height was of term size with lower

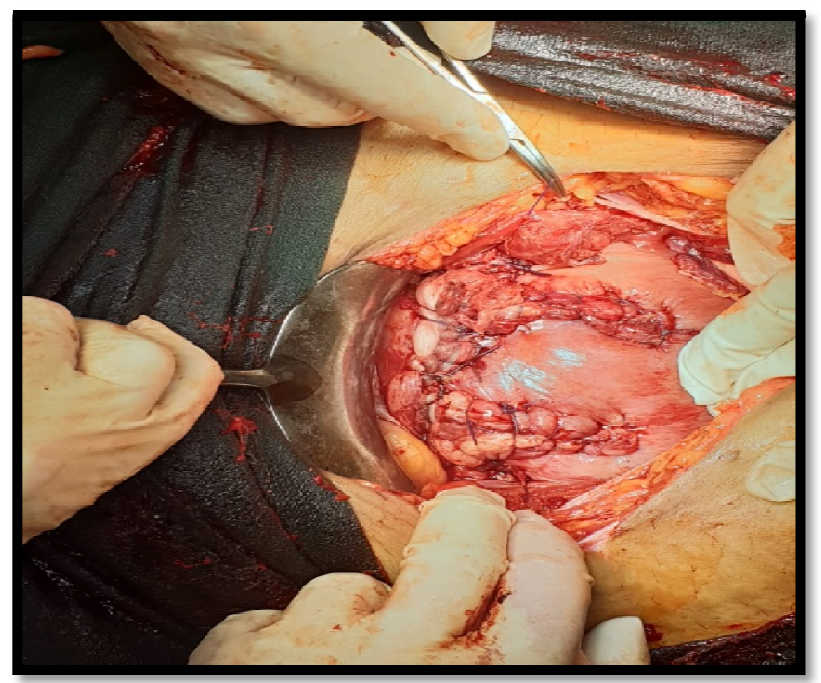

Figure 1: A myoma of about $9 \times 8 \mathrm{~cm}^{2}$ multilobed was removed by giving a nick over the capsule and enucleating it.

pole empty with head felt in right iliac fossa and breech in left. Aseptic dressing was present over the lower abdomen. On auscultation, fetal heart sound was present with a fetal heart rate of 148 beats per minute. The abdomen was tense and tender to touch. Liquor appeared to be clinically adequate with no uterine contractions. Emergency ultrasonography was done to confirm the clinical findings and on ultrasound a large fibroid of $9 \times 8$ $\mathrm{cm}^{2}$ was found to be present on the anterior wall of the lower segment of uterus. The patient was immediately taken for emergency caesarean section after sending the blood for crossmatch and investigations along with arrangement of two units of whole blood as her haemoglobin was $7.6 \mathrm{gm} / \mathrm{dl}$ with haematocrit of $28 \%$.
Abdomen was re-opened from the previous incision site by removing all the sutures and on entering the abdominal cavity, sutures (vicryl) were found over the

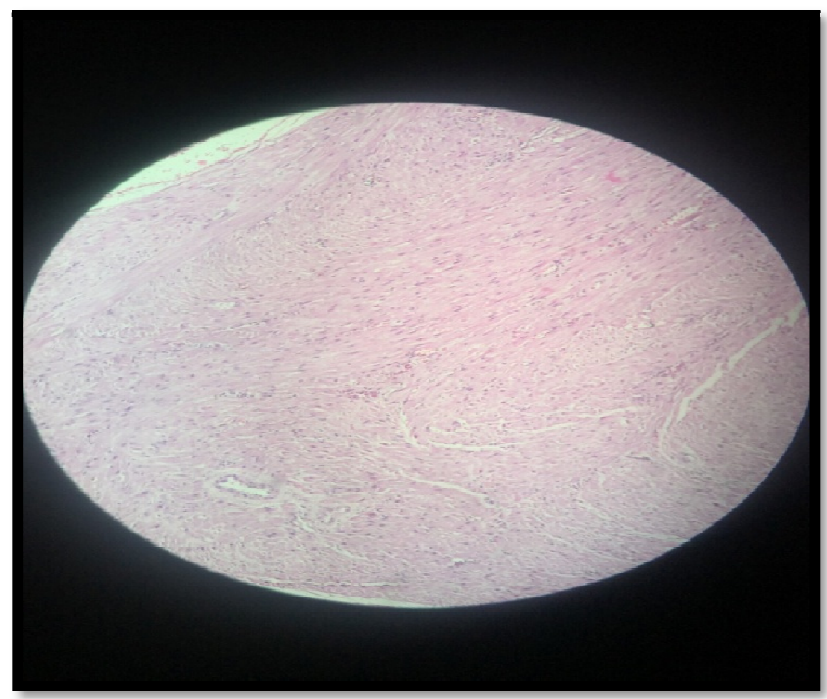

Figure 2: Histopathology showing interlacing bundles of smooth muscle fibres $(\mathrm{H} \& \mathbf{E} \times \mathbf{1 0 0 x})$

anterior surface of uterus, involving the capsule of myoma present over the lower segment of uterus at the incision site. A lateral incision was given on the anterior uterine wall away from the fibroid, to avoid injury to the capsule of myoma and a female baby of $2.9 \mathrm{~kg}$ weight was delivered as breech. Baby cried after tactile stimulation and the APGAR score at 1 and 5 minutes was 7 and 8 respectively. During delivery of the fetus the incision got extended and involved the already incised capsule of myoma and furthermore due to the myoma the two ends of incised uterine wall could not be approximated well, so myomectomy was planned. A myoma of about $9 \times 8 \mathrm{~cm}^{2}$ multilobed was removed by giving a nick over the capsule and enucleating it (Figure 1). Complete haemostasis was achieved by taking multiple sutures at the myoma bed followed by uterine closure in double layers. Another cornual fibroid of about $4 \times 5 \mathrm{~cm}^{2}$ was also present, but it was not removed. Uterus was well retracted at the end of surgery. After achieving complete haemostasis and mops and instrument count, two abdominal drains were kept in situ followed by closure of the abdominal wall in layers. One unit of whole blood was transfused 
The New Indian Journal of OBGYN. 2019 (July-December); 6(1)

intra operatively and another in postoperative period. Patient withstood the procedure well and was shifted to intensive care unit in stable condition. The postoperative period was uneventful with Foley's catheter removed on post-operative day two and both the drains were removed on post-operative day three. All stitches were removed on post-operative day eight and the stitch line was well healed. Histopathological report further confirmed that the specimen sent shows benign tumour with interlacing bundles of smooth muscle fibres arranged in whorls indicating leiomyoma (Figure 2). Patient with her baby was discharged under a satisfactory condition on post-operative day nine and was advised to come for regular follow up after 6 weeks.

\section{Discussion}

Incidence of fibroids during pregnancy varies from $0.1 \%$ to as high as $12.5 \%$. Ultrasound studies have shown that about $20 \%$ of fibroids increase in size and similar percentage decrease during pregnancy ${ }^{1}$. The greatest increase in volume occurs before the 10th gestational week. Different complications with variable rates of incidence have been reported in pregnancy with fibroids which include ante-partum haemorrhage, malposition of the fetus, acute abdomen, laparotomy, red degeneration, intra uterine growth restriction, preterm labour, dysfunctional labour, postpartum haemorrhage, retained placenta, and retained products of conception ${ }^{4,5}$. Because of the increased vascularisation of the uterus during pregnancy, women are at increased risk of bleeding and postoperative morbidity during myomectomy.

Radhika et al conducted a case series of fifteen patients and concluded that pregnancy with fibroids is associated with various adverse events like haemorrhage and should be considered as high-risk pregnancy ${ }^{7}$. Another similar study by Sarwar et al on thirty patients who had pregnancy with fibroid concluded that fibroid in pregnancy, especially multiple intramural fibroids and fibroids larger than $10 \mathrm{~cm}$, cause miscarriage and preterm labour ${ }^{1}$.

\section{Conflict of interest: None. Disclaimer: Nil.}

\section{References}

1.Sarwar I, Habib S, Bibi A, Malik N, Parveen Z. Clinical audit of foeto maternal outcome in pregnancies with fibroid uterus. Journal of Ayub Medical College, Abbottabad. 2012; 24(1): 79-82.

2.Laughlin SK, Baird DD, Savitz DA, Herring AH, Hartmann KE. Prevalence of uterine leiomyomas in the first trimester of pregnancy: an ultrasound-screening study. Obstetrics and gynecology. 2009; 113(3): 630-35.

3.Akhtar N, Sulthana S, Zabin F. Successful Outcome of pregnancy with large fibroid uterus- a case report. Bangladesh J Obstet Gynaecol. 2010; 25(2): 87-9.

4.Koike T, Minakami H, Kosuge S, Usui R, Matsubara S, Izumi A, et al. Uterine leiomyoma in pregnancy: its influence on obstetric performance. The journal of obstetrics and gynaecology research. 1999; 25(5): 309-13.

5.Lev-Toaff AS, Coleman BG, Arger PH, Mintz MC, Arenson RL, Toaff ME. Leiomyomas in pregnancy: sonographic study. Radiology. 1987;164(2): 375-80.

6.Ciavattini A, Clemente N, Delli Carpini G, Di Giuseppe J, Giannubilo SR, Tranquilli AL. Number and size of uterine fibroids and obstetric outcomes. The journal of maternalfetal \& neonatal medicine. 2015; 28(4): 484-8.

7.Radhika BH, Naik K, Shreelatha S, Vana H. Case series: pregnancy outcome in patients with uterine fibroids. J Clin Diagn Res. 2015; 9: QR01-4.
Ashu Yadav ${ }^{1}$, Naina Kumar ${ }^{2}$
${ }^{1} P G 2^{\text {nd }}$ year, Department of Obstetrics and Gynaecology, Maharishi Markandeshwar Institute of Medical Sciences \& Research, Mullana, Haryana, India; ${ }^{2}$ Associate Professor, Department of Obstetrics and Gynaecology, All India Institute of Medical Sciences, Mangalagiri, Guntur, India. 